\title{
Fluorine-19 MRI for detection and quantification of immune cell therapy for cancer
}

\author{
Fanny Chapelin ${ }^{1}$, Christian M Capitini ${ }^{2^{*}}$ and Eric T Ahrens ${ }^{3^{*}}$
}

\begin{abstract}
Over the past two decades, immune cell therapy has emerged as a potent treatment for multiple cancers, first through groundbreaking leukemia therapy, and more recently, by tackling solid tumors. Developing successful therapeutic strategies using live cells could benefit from the ability to rapidly determine their in vivo biodistribution and persistence. Assaying cell biodistribution is unconventional compared to traditional small molecule drug pharmacokinetic readouts used in the pharmaceutical pipeline, yet this information is critical towards understanding putative therapeutic outcomes and modes of action. Towards this goal, efforts are underway to visualize and quantify immune cell therapy in vivo using advanced magnetic resonance imaging (MRI) techniques. Cell labeling probes based on perfluorocarbon nanoemulsions, paired with fluorine19 MRI detection, enables background-free quantification of cell localization and survival. Here, we highlight recent preclinical and clinical uses of perfluorocarbon probes and ${ }^{19} \mathrm{~F}$ MRI for adoptive cell transfer (ACT) studies employing experimental T lymphocytes, NK, PBMC, and dendritic cell therapies. We assess the forward looking potential of this emerging imaging technology to aid discovery and preclinical phases, as well as clinical trials. The limitations and barriers towards widespread adoption of this technology, as well as alternative imaging strategies, are discussed.
\end{abstract}

Keywords: ${ }^{19} \mathrm{~F}$ MRI, Fluorine-19, Perfluorocarbon, Immunotherapy, Adoptive cell transfer, Cancer, T cell

\section{Background}

Surgery, chemotherapy and radiotherapy have been used for decades as primary strategies against cancer in patients [1]. However, non-specific toxicities to healthy cells and life threatening side effects from chemotherapy and radiation, as well as drug and radiation cancer cell resistance, have motivated investigators to seek new treatment approaches to improve curative outcomes and quality of life. Immunotherapeutic strategies have emerged as a fourth pillar for cancer treatment, which holds promise for less toxic side effects and durable response rates against residual primary cancers and metastases, even if tumors were previously considered chemorefractory.

\footnotetext{
* Correspondence: ccapitini@pediatrics.wisc.edu; eta@ucsd.edu

${ }^{2}$ Department of Pediatrics and Carbone Cancer Center, University of Wisconsin School of Medicine and Public Health, 1111 Highland Avenue, Madison, WI 53705, USA

${ }^{3}$ Department of Radiology, University of California of San Diego, 9500 Gilman Dr. \#0695, La Jolla, CA 92093-0695, USA

Full list of author information is available at the end of the article
}

Throughout life, the immune system actively prevents neoplastic development through immunosurveillance [2]. The innate immune system, including monocytes, macrophages, dendritic cells (DCs) and natural killer (NK) cells, provide front line protection through cancer cell recognition, lysis, and pro-inflammatory cytokine production [3]. T and $\mathrm{B}$ cells, main effectors of the adaptive immune system, mediate antigen-specific responses against cancer and can form long term memory [4]. Nonetheless, cancer cells have evolved mechanisms to evade such surveillance, such as MHC downregulation and cytokine secretion, to create an immunoprivileged microenvironment [5]. Adoptive cell therapy (ACT) aims to counterbalance this effect by providing highly activated effector cells into the body. Early treatments developed by Rosenberg et al., comprised of T cells derived from the tumor-bearing host, are referred to as tumor-infiltrating lymphocytes (TILs) [6]. Subsequently, complex in vitro engineering of the T cell receptor (TCR) by gene transfer, as well as de novo MHC-independent

(c) The Author(s). 2018 Open Access This article is distributed under the terms of the Creative Commons Attribution 4.0 International License (http://creativecommons.org/licenses/by/4.0/), which permits unrestricted use, distribution, and 
targets called Chimeric Antigen Receptors (CAR) were developed [7]. Progress in the design of CARs included optimization of antigen specificities, $\mathrm{T}$ cell activation mechanisms, effector function and $\mathrm{T}$ cell persistence [8]. Over 300 clinical trials are currently investigating TILs, TCR and CAR T cell therapies [9].

Inherent in the mind's eye of clinical investigators is that cell trafficking behavior in vivo may be predictive of therapeutic outcomes. For example, in CAR T cell trials against solid tumors [10], basic assumptions are that therapeutic cell survival and trafficking to the tumor sites are required for a putative therapeutic effect. Clinicians are currently blinded as to whether cells reach their desired tissue targets. Effector cell proliferation and enzyme production is another avenue for assaying ACT activity [11]. Overall, surrogate biomarkers capable of visualizing and quantifying sites harboring cells in vivo, as well as survival of ACT at tumor and lymphoid organs, would be invaluable for predicting therapeutic response following administration. Indeed the Food and Drug Administration (FDA) is interested in expanding non-invasive imaging platforms of tracking cells to aid in safety monitoring [12]. In 2008, the Cell, Tissues and Gene Therapies Advisory Committee of the FDA Center for Biologics Evaluation and Research stated that sponsors should be encouraged to develop real-time imaging/ labeling methods for tracking cells [13]. Non-invasive clinical imaging techniques including Magnetic Resonance Imaging (MRI) and nuclear imaging are candidates for developing real-time, quantitative biomarkers for ACT $[14,15]$.

In 2010, the FDA's Center for Devices and Radiological Health started an initiative to reduce unnecessary radiation exposure from medical imaging [16]. MRI can provide anatomical and disease diagnostic information with intrinsic soft tissue contrast without ionizing radiation. Shortly after the invention of proton MRI, the feasibility of fluorine-19 $\left({ }^{19} \mathrm{~F}\right)$ MRI was demonstrated in 1977 by Holland et al. [17]. ${ }^{19} \mathrm{~F}$ is a natural halogen, non-radioactive isotope of fluorine. ${ }^{19} \mathrm{~F}$ has a relative sensitivity of $83 \%$ compared to ${ }^{1} \mathrm{H}$ and essentially devoid in biological tissues of interest [18], providing background-free imaging of ${ }^{19} \mathrm{~F}$-based probes. A description of ${ }^{19} \mathrm{~F}$ MRI physics can be found elsewhere [19]. Fluorine-dense perfluorocarbon (PFC) nanoemulsions have been specifically engineered to be endocytosed, even by non-phagocytic cells in culture [20]. After cell inoculation, ${ }^{19} \mathrm{~F}$ MRI signal intensity is linearly proportional to ${ }^{19} \mathrm{~F}$-atom concentration, enabling unbiased measurements of apparent cell numbers from images [21].

Here, we provide a brief overview of current and emerging experimental strategies to detect ACT using ${ }^{19} \mathrm{~F}$ MRI. We focus on the characterization of ACT immune cell populations labeled with PFC nanoemulsions including $\mathrm{T}$ cells, $\mathrm{NK}$ cells and $\mathrm{DC}$ vaccines. We describe how this approach can benefit the discovery and preclinical phases of the therapeutic development and potentially clinical trials.

\section{PFC-based nanoemulsion probes}

PFC molecules have properties that are attractive for cell labeling and ${ }^{19} \mathrm{~F}$ MRI tracking applications [22]. Their strong C-F covalent bonds render them chemically inert and are not metabolized in vivo [23]. Moreover, PFCs often display simultaneous lipo- and hydro-phobic properties [24] and do not dissolve in cell membranes. PFCs commonly used for ${ }^{19} \mathrm{~F}$ MRI imaging include perfluoropolyether (PFPE), perfluoro-15-crown-5-ether (PCE) and perfluorooctyl bromide (PFOB) [22]. PFPE and PCE are linear and cyclic polymers, respectively, each with numerous chemically-equivalent fluorine yielding high MRI sensitivity. PFOB has less MRI sensitivity overall due to chemically inequivalent F-sites [25].

Neat PFC materials are dense oils. Emulsification is used to make a colloidal suspension of the PFC oil that is stabilized using a surfactant. The surfactant coat can also impart desirable surface properties that promote cell uptake in culture $[26,27]$. The most commonly used classes of surfactants are pluronics and phospholipids [28]. Key design considerations in nanoemulsion formulation include a small droplet size (typically 100$200 \mathrm{~nm}$ ), a narrow size range (e.g., polydispersity index $<0.2)$ and a high fluorine concentration $(\sim 20-30 \% v / \mathrm{v})$ to minimize volume added to culture. Nanoemulsion formulations may also be complexed with fluorophores, for example near infrared dyes, to create 'dual-mode' agents [20, 22, 29]. Recent reviews exhaustively cover PFC nanoemulsion design [22, 30].

Different published studies use a range of emulsion particle sizes [20,31]. The mean emulsion droplet size can impact the cell labeling process [32]. Larger oil droplets $(>200 \mathrm{~nm})$ are effective in labeling flask-adherent cells, such as DCs, where successful wash steps can be implemented and can potentially result in higher overall labeling levels [31]. However, a smaller droplet size $(<180 \mathrm{~nm})$ allows excess agent not taken up by suspended cells, such as lymphocytes, to be discarded with the supernatant during wash. Emulsion production ideally yields a homogenous size distribution, which is easier to achieve with smaller droplet sizes. Unintended, outlying large droplets ('stability demons') may evade detection in dynamic light scattering particle size measurements of the batches. These demons can lead to emulsion instability over time [33] and may spin-down with the cells. Overall, in properly designed experiments, free residual emulsion in the cell inoculant is de minimis and inconsequential in view of detection limits of the MRI technique. 


\section{Immune cell labeling}

Cell labeling in culture is generally performed by simple co-incubation with PFC as another factor in the media, followed by a wash step. Labeling periods range from several hours [21, 34-36] to a day or more [37-39] to allow for endocytic uptake to occur. Determinants of obtainable PFC cell uptake include (i) dose of PFC in media, (ii) cell cytoplasmic volume and (iii) phagocytic properties of cells. Typically, several concentrations and incubation times are tested to optimize uptake while minimizing potential cell viability and phenotype alterations [20].

Lymphocyte labeling can be challenging due to their small cellular and cytoplasmic size that limits the number of nanoemulsion droplets it can hold. In addition, lymphocytes are not naturally phagocytic. Optimal labeling efficiency is attained when cells are in log phase of division. PFC uptake will follow a dose response in the shape of a sigmoidal curve [39]. A critical factor for strong labeling of lymphocytes is that the culture must be viable and actively expanding, typically aided by aggressive cytokine and co-stimulatory molecule engagement (e.g., irradiated 4-1BBL/IL-15 expressing feeder cells, CD3/CD28 beads, etc.) as discussed elsewhere [37, 40]. Preferred PFC nanoemulsion formulations enable labeling of lymphocytes for in vivo tracking without the use of transfection agents [20], as shown in preclinical studies [22, 41] (Table 1). In contrast, macrophages and immature DCs possess a larger cytoplasmic volume and are aggressively phagocytic [42] and thus are more readily labeled to higher levels.

After washes, cell labeling levels can be measured in a pellet sample using conventional ${ }^{19} \mathrm{~F}$ nuclear magnetic resonance (NMR) spectroscopy to yield the mean ${ }^{19} \mathrm{~F} /$ cell. Various cell microscopy methods have been used to validate intracellular compartmentalization of PFC droplets. Using transmission electron microscopy, the emulsion droplets appear as electron-sparse ovoids against counterstain [31, 43, 44]. Emulsion droplets often coalesce into encapsulated vesicles consistent with lysosomal storage in lymphoid-type and stem cells [45]. In the case of antigen presenting cells (APCs, e.g., DCs), PFC traffics to more specialized compartments, such as macropinosomes [43].

Dual-mode, PFC-fluorescence nanoemulsions [20] enable flow cytometry of labeled cells, as well as optical microscopy in histology sections. Confocal microscopy images of labeled immune cells clearly show intracellular localization (Figs. 1a-b). PFC localization is inconsistent with dominate cell surface labeling, which has been confirmed by explicit cell membrane staining (Fig. 1a-b) and by cellular proliferation dyes such as 5(6)-Carboxyfluorescein N-hydroxysuccinimidyl ester (CFSE, Fig. 1c). Detailed fluorescent microscopy studies using a dual-mode emulsion with a $\mathrm{pH}$ sensitive dye confirmed that the PFC emulsion traffics into low-pH (lysosomal) vesicles over time [45]. This intracellular compartmentalization is the steady-state in living cells, as the PFC is not degraded in the cell and there is no evidence for active exocytosis [45].

Cell labeling should not alter cell viability, proliferation, phenotypic markers, or function, as described in several reports $[46,47]$. In a recent study, Chapelin et al. performed in vitro studies in human CAR $\mathrm{T}$ cells showing that PFC labeling does not alter cell viability, division rate and phenotype (defined by CD4/CD8 expression) for at least 14 days post-labeling. Similarly, NK cells labeled with PFPE nanoemulsion exhibited unaltered viability and phenotype [37]. Somanchi et al. published a detailed protocol for expansion and PFPE labeling of NK cells [36]. Cytotoxicity of labeled NK cells against cancer cells in vitro was comparable to non-labeled cells, and cytokine and perforin secretion was preserved [36, 37] (Table 1). The most detailed in vitro study to date involved PFC-labeled primary human DCs [39]; cells were assayed for viability, maturation phenotype, cytokine production, $\mathrm{T}$ cell stimulatory capacity, and chemotaxis [39], and no differences in these parameters were observed between labeled and unlabeled cells [39].

\section{T cells}

Adoptive $\mathrm{T}$ cell therapy can elicit sustained tumor-specific killing in vivo and has the potential to form long-term memory against tumor-associated antigens. Fundamental questions remain to be answered regarding $\mathrm{T}$ cell biodistribution, anti-cancer activity and persistence after infusion. First, non-invasive cell tracking methods could assist in optimizing delivery method (systemic versus local) and dosage. ACT homing to solid tumors remains a challenge, and tracking methods could further our understanding of the factors affecting tumor homing, which may be predictive of response to therapy [48, 49]. Additionally, evaluation of the impact of co-therapies, such as checkpoint inhibitors, by ${ }^{19} \mathrm{~F}$ MRI could yield insights into the role of adjuvant treatments on $\mathrm{T}$ cell behavior.

In preclinical studies, after infusion of PFC-labeled immune cells, one approach for quantitative biodistribution assessment is via conventional ${ }^{19} \mathrm{~F}$ NMR spectroscopy of intact, fixed tissues samples (i.e., NMR cytometry) [40, 50]. NMR cytometry has the advantage of rapid sample throughput with sensitivity limits of detection of order $10^{3} \mathrm{~T}$ cells per sample [40]. In a recent NMR cytometry study, CAR $\mathrm{T}$ cells targeting glioma tumors expressing EGFRvIII [40] (Table 1) were labeled with PFC emulsion overnight and subsequently injected IV. Panel necropsy at several time points post-infusion followed by ${ }^{19} \mathrm{~F}$ NMR measurement of organ fluorine content yielded the apparent transferred cell number in each tissue (Fig. 1d). On average, twice as many CAR T-cells homed to the tumor 


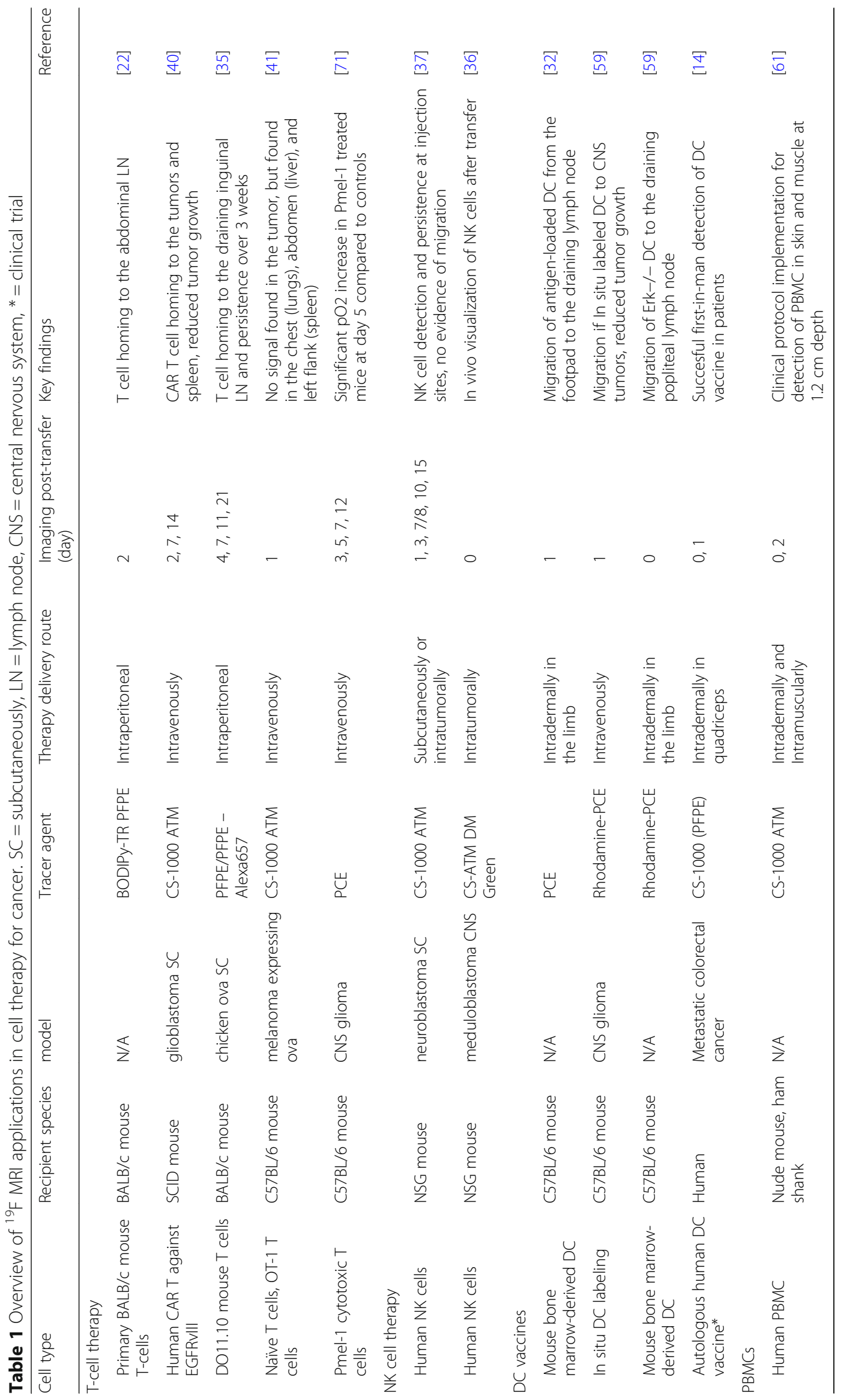



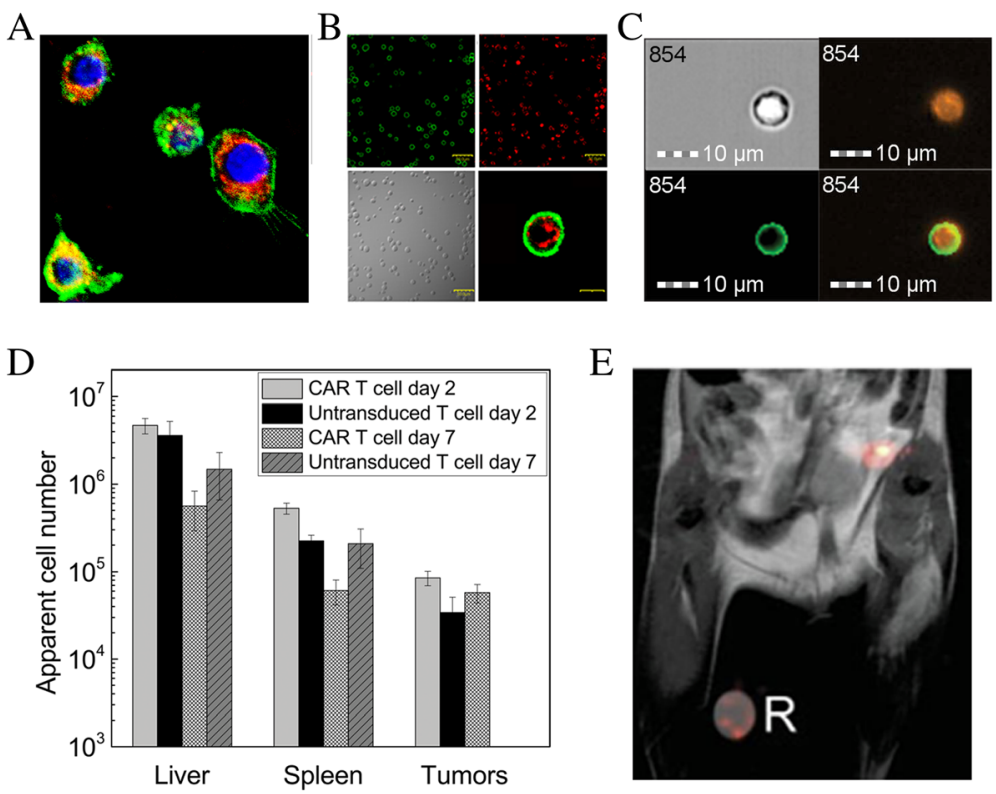

Fig. 1 Immune cells labeled with PFC and in vivo distribution. a Murine DCs labeled with dual-mode BODIPY- ${ }^{19} \mathrm{~F}$ PFC nanoemulsion as seen in fluorescent micrographs of the cytoplasm (red), along with Hoechst labeled nuclei (blue) and the CD45-FITC labeled cell surface (green). b Murine primary activated T cells labeled with dual-mode PFC nanoemulsion showing cytoplasmic localization of CD4-FITC labeled cell surface (green, upper left), the PFC nanoemulsion (red, upper right), white-light image of labeled T cells (lower left) and fusion image of CD4-FITC-PFC (lower right). Scale bar is $20 \mu \mathrm{m}$. c NK cells isolated from a Balb/c spleen and incubated with a dual-mode PFC agent (BODIPY $\left.-{ }^{19} \mathrm{~F}\right)$ for $24 \mathrm{~h}$, then incubated with CFSE for 15 min. Upper left: Darkfield microscopy of a Balb/c NK cell. Upper right: BODIPY $-{ }^{19} \mathrm{~F}$ (orange) is seen in the entire cell. Lower left: CFSE (green) is taken up in the cell membrane. Lower right: Fusion image showing labeling with BODIPY ${ }^{19} \mathrm{~F}$ and CFSE. Scale bar is $10 \mathrm{~mm}$. d Biodistribution quantification of fixed tissue samples by ${ }^{19} \mathrm{~F}$ NMR 2 or 7 days after human CAR T cell treatment in subcutaneous glioma (U87-EGFRvIII) bearing SCID mice. e ${ }^{1} \mathrm{H} /{ }^{19} \mathrm{~F}$ overlay MRI showing PFPElabeled antigen specific $T$ cells in the draining lymph node of a BALB/c mouse locally injected with chicken ova. $R$ indicates a reference capillary used for quantification. (Figure adapted from References [22, 35, 40])

and spleen compared to naïve T cells. In addition, CAR T cell persistence surpassed that of naïve T cells [40]. Cell quantification in this study did not account for T cell division in vivo. The CAR T cell treatment resulted in significant tumor growth decline and correlated to the number of cells homing to the tumor and spleen.

$\mathrm{T}$ cell distribution can also be monitored by ${ }^{19} \mathrm{~F}$ MRI in vivo cytometry. In early studies, Srinivas et al. [35] labeled antigen-specific DO11.10 mouse T cells with PFC emulsion and infused them into a BALB/c host receiving a local injection of ovalbumin with adjuvant [35] (Table 1). The study tracked the dynamic accumulation and clearance of labeled $\mathrm{T}$ cells in the lymph node proximal to the antigen injection site (Fig. 1e). ${ }^{19} \mathrm{~F}$ MRI allowed for T cell imaging and quantification up to 3 weeks post-transfer. Gonzales et al. [41] used a similar approach in a mouse B16 Ova melanoma tumor model (Table 1). The melanoma cell line was engineered to express Ova and tested using infused PFC-labeled splenocytes, naïve T cells and Ova-peptide activated $\mathrm{T}$ cells in vivo. ${ }^{19} \mathrm{~F}$ MRI images displayed bright hot-spots corresponding to splenocyte and $\mathrm{T}$ cell distribution to the lungs, liver and spleen; no cells were detected in tumor by MRI, but could be detected in small numbers by flow cytometry. These results corroborate NMR studies [40] (Table 1).

\section{NK cells}

Another ACT strategy involves infusing NK cells, which are key effectors of innate immunity and by definition not antigen specific. NK cells contribute to cancer immuno-surveillance. They screen local cells in situ and recognize cancer cells expressing altered MHC molecules or downregulated MHC expression, or antibodycoated tumor cells, leading to NK cell perforin release and cancer cell death [51]. Similarly to T cells, NK cell therapies are usually administered intravenously, but also intratumorally $[52,53]$. Because NK cells cannot form memory, knowledge of NK cell activity and persistence will be critical to better understand the need for repeated infusions and to develop 'smarter' cell delivery methods for solid tumors.

Bouchlaka et al. reported that PFC-labeled human NK cells were detectable by longitudinal MRI up to 8 days after intratumoral injection in NSG mice [37] (Fig. 2a). 

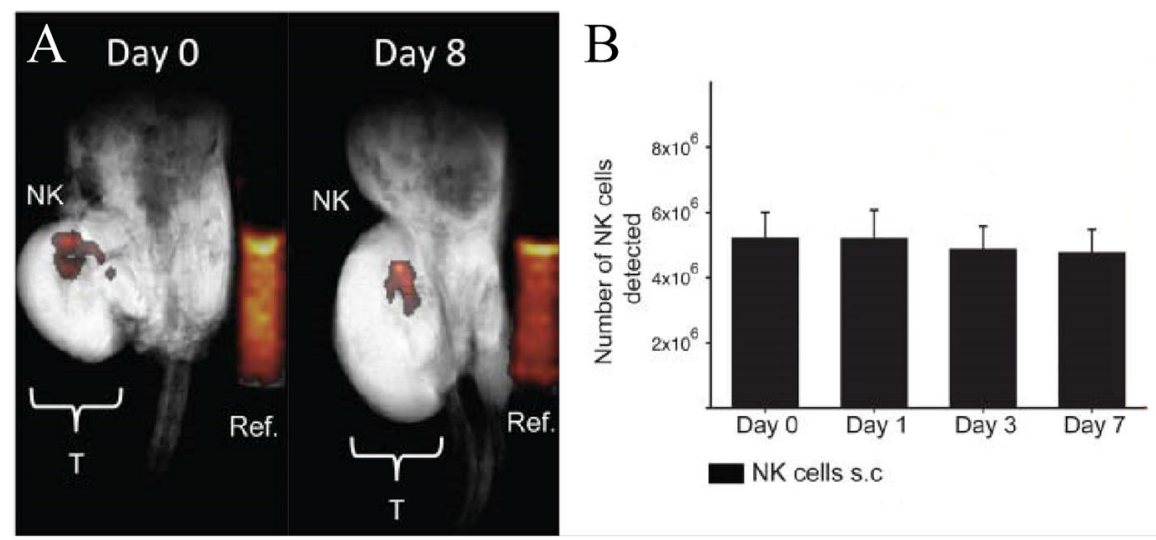

NK cells s.c

Fig. 2 NK cells in mice. a In vivo composite ${ }^{1} \mathrm{H} /{ }^{19} \mathrm{~F}$ MRI images of ${ }^{19} \mathrm{~F}$-labeled human NK cells at day 0 and day 8 post NK therapy in NSG mice bearing human xenograft tumors (Ref. is external quantification reference tube, and "T" is tumor). b Mean number of NK cells detected at the tumor site is denoted for each imaging time point. The number of NK cells is stable over a week. (Adapted with permission from Reference [37])

NK cell number remained relatively stable over 1 week (Fig. 2b). When NKs were injected subcutaneously, NK cell number at the injection site decreased over the same time period and migrated to tumor as evidenced by a reduction in tumor size, although there were too few cells to detect them within the tumor by MRI. NK cells may have insufficient anti-tumor activity and fail to persist in vivo [54]. To palliate such effects, researchers are now incorporating CARs into NK cells, thereby providing antigen-specificity and potentially better anti-tumor activity, with unknown effects on NK persistence [55]. ${ }^{19} \mathrm{~F}$ MRI may be useful for the development of next generation NK therapies.

\section{Dendritic cells}

In vivo cytometry was originally described and experimentally tested to visualize DCs in mice [43]. DCs are professional APCs that form the link between innate and adaptive immunity. DCs modulate the inflammatory response by precisely activating $\mathrm{T}$ cell subtypes such as helper and cytotoxic T cells. DCs are often administered intradermally to facilitate their entry into lymphatic vessels. Therapeutic DCs are usually primed with specific tumor antigens prior to injection to enhance specific antigen presentation and chemokine production [56, 57]. In one study, 'theranostic' PFC nanoemulsions were created for one-step DC labeling and tumor priming with antigen [58]. Labeled DCs were injected intradermally, and ${ }^{19} \mathrm{~F}$ MRI $18 \mathrm{~h}$ post-transfer showed DC migration lines toward the draining lymph node [31] (Table 1). In a different study, PFC-labeled mature human DCs were also shown to migrate from a NOD/ SCID mouse thigh subcutaneous injection site to the draining popliteal lymph node within $18 \mathrm{~h}$ of injection [39]; immature DCs, on the contrary, did not leave the injection site. $\mathrm{Ku}$ and coworkers used an in situ cell labeling approach, where PFC nanoemulsion was injected intradermally and taken up by resident DCs, in an effort to visualize DCs migrating into GL261 CNS glioma tumors [59] (Table 1). Injection of rhodamine-conjugated PFC nanoemulsion in either wild type or $\mathrm{Erk}^{-1-} \mathrm{C} 57 \mathrm{BL} / 6$ mice showed greater fluorine labeled DCs migrating into tumor tissue of $\mathrm{Erk}^{-/-}$ C57BL/6 mice and as a result, slower tumor growth. When labeled ex vivo with the same PFC agent, $\mathrm{Erk}^{-/-} \mathrm{DCs}$ injected intradermally were shown to migrate further towards the popliteal lymph node compared to wild type DCs by ${ }^{19} \mathrm{~F}$ MRI. Ex vivo ${ }^{19} \mathrm{~F}$ NMR cytometry of excised
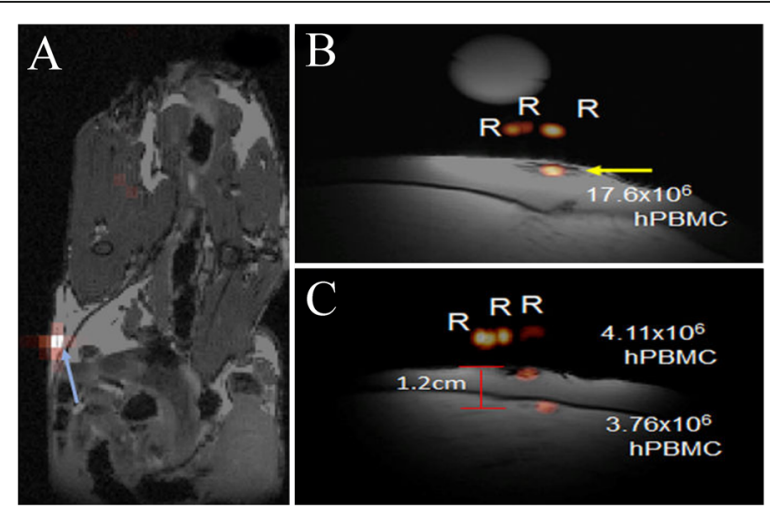

Fig. 3 PBMC ${ }^{19} \mathrm{~F} \mathrm{MRI} \mathrm{imaging} \mathrm{in} \mathrm{immunocompromized} \mathrm{mice} \mathrm{and}$ phantoms. a In vivo composite ${ }^{1} \mathrm{H} /{ }^{19} \mathrm{~F}$ MRI image of PFPE-labeled human PBMC following subcutaneous flank injection of $6 \times 10^{6}$ cells (blue arrow) in nude mouse. For preliminary clinical MRI protocol implementation, PFPE-labeled PBMC were injected intradermally and intramuscularly in a ham shank phantom. $\mathbf{b}$ Intradermal injection alone consisted of $20 \times 10^{6}$ cells (yellow arrow). c Composite images of shanks receiving both intradermal and intramuscular PBMC injections of $4.5 \times 10^{6}$ cells each. $R$ indicate references used for quantification. (Adapted from Reference [61]) 
lymph nodes quantitatively correlated to the MRI findings. Fluorine labeling may therefore help elucidate regulators of DC migration and enable optimization of DC vaccine therapies.

\section{Peripheral blood mononuclear cells}

PBMC vaccines encompass both effector cells, (such as $\mathrm{T}$ and NK cells) and professional APCs (B cells, monocytes and DCs). Vaccines prepared from PBMCs are FDA-approved for prostate cancer treatment [60]. Fink et al. [61] investigated the use of PFC agents to label human PBMC samples from patients to enable in vivo detection (Table 1). The authors showed that all PBMC cells label, but to varying degrees, and uptake measurements in sorted cell subtypes yielded a labeling $\left({ }^{19} \mathrm{~F} / \mathrm{cell}\right)$ profile. When injected in nude mice flanks, PBMC could be detected by ${ }^{19} \mathrm{~F}$ MRI 2 hours and 2 days post-injection (Fig. 3a). To optimize clinical ${ }^{19} \mathrm{~F}$ MRI protocols for PBMC vaccine imaging in patients, the authors injected PFC-labeled human PBMC in ham shanks. Both intradermal (Fig. 3b) and intramuscular (Fig. 3c) PBMC injections were detected by clinical $3 \mathrm{~T}$ MRI using a custom surface coil at high sensitivity with a detection limit of $\sim 6 \times 10^{4}$ PBMC.

\section{Intracellular oximetry as a biomarker for cancer immunotherapy}

An intrinsic property of PFCs is that they display weak molecular cohesion, enabling gas dissolution [24]. In fact, extensive work was conducted in the late 1990's [62, 63] to emulsify PFCs into biocompatible, excretable, and readily injectable blood substitutes to address hospital blood shortages [64]. Building on in vivo cytometry technology, a logical extension is to exploit known bio-sensing properties of the PFC molecules inside the cell. Specifically, certain PFC molecules readily coordinate paramagnetic oxygen, which shortens the ${ }^{19} \mathrm{~F}$ spin-lattice relaxation time
$\left(T_{1}\right)$, where $T_{1}$ varies linearly with the absolute partial pressure of oxygen $\left(\mathrm{pO}_{2}\right)$ [65]. $\left(\mathrm{T}_{1}\right.$ is the characteristic time constant for the ${ }^{19} \mathrm{~F}$ nuclei to align along the MRI's magnetic field, on the order of 0.5 to $2 \mathrm{~s}$.) PFC emulsions have previously been used to measure $\mathrm{pO}_{2}$ in vivo using MR techniques [66-69]. However, a novel use of ${ }^{19} \mathrm{~F}$-based cell tracking is to use ${ }^{19} \mathrm{~F} \mathrm{~T}_{1}$ measurements to monitor intracellular oximetry. The first study using in vivo cytometry to investigate cancer cell $\mathrm{pO}_{2}$ changes in response to therapy was performed in a $9 \mathrm{~L}$ rat model of brain glioma [70]. Authors showed that treatment with chemotherapy (BCNU) induced a significant and sustained $\mathrm{pO}_{2}$ increase in the labeled cancer cells. A follow-up study used a similar approach to monitor intracellular oxygen changes of murine GL261 glioma cells in response to Pmel-1 cytotoxic $\mathrm{T}$ cells [71] (Table 1). Labeled glioma cells appear as a background-free hotspot overlaid on a proton image (Fig. 4a). A voxel (volume element) encompassing the hotspot is delineated, and MRI spectroscopy methods yield the voxel $R_{1}=1 / T_{1}$ (Fig. 4b); absolute $\mathrm{pO}_{2}$ is then calculated from a calibration curve. MRI results correlated to histopathology analysis, confirming small numbers $\left(\sim 10^{3}\right)$ of infiltrating cytotoxic T cells in the tumor region. These studies demonstrate the feasibility of using in vivo cytometry for real-time, cell-specific oximetry as an early biomarker of anti-cancer responses before MRI-visible tumor shrinkage is observed.

\section{Limitations of PFC labeled cells}

Generally, with PFC labeled cells having a mitotic phenotype, cell division and subsequent dilution of the intracellular label can potentially limit long-term studies and decrease the accuracy of cell quantification [40]. There is no evidence for active exocytosis or degradation of the PFC droplets once internalized by viable cells. Death of labeled cells leads to dispersion of the reagent
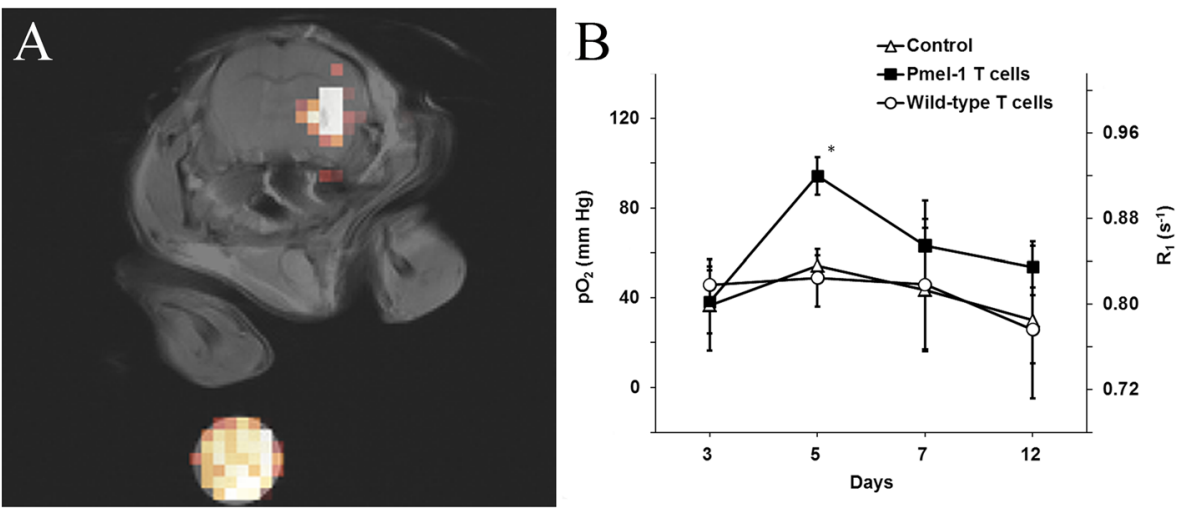

Fig. 4 Indirect visualization of T cell therapy efficacy via cancer cell oximetry. a Composite ${ }^{19} \mathrm{~F}$ and ${ }^{1} \mathrm{H}$ image of PCE labeled glioma (GL261) cells in the right striatum 5 days after tumor inoculation in C57BL/6 mice. A diluted PCE reference capillary is placed below the animal (bottom). $\mathbf{b}$ In vivo longitudinal tumor $\mathrm{pO}_{2}$ measurement after Pmel-1 mouse derived CD8+ T cell, wild-type T cell injection or no treatment. Transient hyperoxia is observed with administration of Pmel-1 CD8+ T cells. (Adapted from Reference [71]) 
and thus a loss of ${ }^{19} \mathrm{~F}$ signal. Potentially, the PFC droplets can also be transferred to macrophages that have engulfed dead cells; if a large number of these macrophages remain in a region of interest, quantification accuracy may suffer. Importantly, the ${ }^{19} \mathrm{~F}$ signal values clearly diminish at cell injection sites over time if the cells are apoptotic, and this cell loss is accurately quantifiable in longitudinal scans [14, 72], which is an advantage over prior-art iron-oxide nanoparticle based cell tracking approaches [73, 74]. Ultimately, clearance of PFC agents from the body occurs via uptake by cells of the RES, particularly Kupffer cells of the liver, followed by lung exhalation [75]. In fact, the ${ }^{19} \mathrm{~F}$ liver signal, and the effective number of cells represented by this value, can be used as a proxy to calculate the dead fraction of the infused cell product [40].

\section{Cell sensitivity}

Since its introduction in clinical practice in the 1980s, MRI has experienced remarkable growth and development. But implementation of new clinical applications comes with challenges both technical and logistical in nature. Often a key limitation of ${ }^{19} \mathrm{~F}$ MRI probes is sensitivity. Unlike conventional ${ }^{1} \mathrm{H}$ MRI, where the probe (water) concentration $\left(>100\right.$ Molar $\left.{ }^{1} \mathrm{H}\right)$ and thus sensitivity is high, ${ }^{19} \mathrm{~F}$ MRI is limited by the total amount and distribution of fluorine atoms introduced into the subject's tissue. The limits of detection using ${ }^{19} \mathrm{~F}$-based imaging ranges from $\sim 10^{3}$ to $\sim 10^{5}$ cells per voxel [76]. For a given experiment, results depend on specific details, such as the PFC molecule and nanoemulsion used, the cell type (i.e., cell cytoplasm size) labeled, viability of cell culture and commensurate label uptake, image acquisition methods, magnetic field strength, and MRI detector configuration $[40,46,61,72]$. Looking forward, there are multiple, clinically-feasible, technical avenues for improving cell detection sensitivity that are vigorously being investigated involving new probe design and data acquisition methods $[30,77,78]$.

\section{Future clinical perspective}

${ }^{19} \mathrm{~F}$ MRI cell detection techniques are just beginning to be employed in clinical trials (Table 1), and feasibility has been established in a first-in-human clinical study [14]. An autologous DC vaccine was labeled with a PFC nanoemulsion ex vivo and re-injected into colorectal cancer patients intradermally (Fig. 5a). ${ }^{19} \mathrm{~F}$ MRI enabled visualization of injected DCs at the injection site and longitudinal persistence evaluation (Fig. 5b).

When engaging cell therapy regulatory agencies, such as the US FDA, safety is the primary concern. Within the FDA, ${ }^{19} \mathrm{~F}$ labeled therapeutic cells are considered a combination product and regulated by the Center for Biologics Evaluation and Research (CBER). Generally, PFC is viewed as having a favorable safety profile and is used in several FDA-approved medicines [79], as well as for contrast-enhanced ultrasound [80]. For cellular therapies, the release criteria for PFC labeled cell batches should match the release criteria expected for the unlabeled cell product [14], such as total nucleated cell count, cell viability, Gram stain, bacterial contamination and endotoxin levels.

Post-infusion, cell viability and anti-tumor efficacy of PFC labeled cells may also be examined in preclinical studies as part of the investigational new drug (IND) application for the cell therapy product. However, imaging results in rodent models of cellular immunotherapy can have significant limitations and may not well reflect how the cell product will behave in patients. Besides the obvious immunological dissimilarities, particularly with immunodeficient xenograft models, typical total cell number doses infused in ACT trials are vastly higher in human trials compared to mice $\left(\sim 10^{10}\right.$ versus $10^{6}$, respectively). Dosing on a cell number/kg basis can help
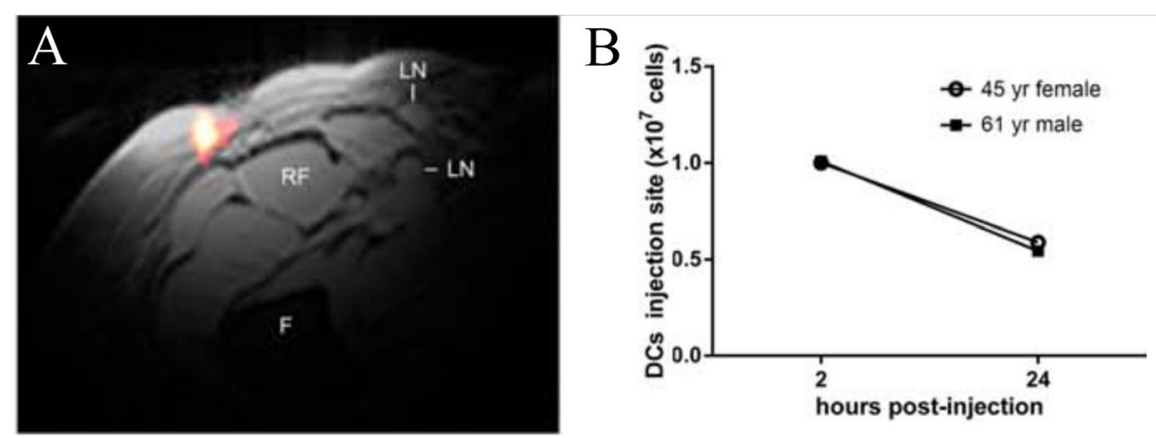

Fig. 5 Clinical DC vaccine imaging following intradermal administration in patients with colorectal cancer. a In vivo composite ${ }^{1} \mathrm{H} /{ }^{19} \mathrm{~F}$ MRI image of $\left(10^{7}\right)$ PFPE-labeled autologous DCs $4 \mathrm{~h}$ after intradermal injection in a 53-year-old female patient ( $F=$ femur, $R F=$ rectus femoris, $L N=$ inguinal lymph node). b Quantification of apparent DC numbers using the in vivo ${ }^{19} \mathrm{~F}$ MRI data, measured in two patients. At $24 \mathrm{~h}$ post-inoculation, half of the injected DCs are detected at the injection site. (Adapted from Reference [14]) 
predict translation to clinical dosing. However, because tumor size may be of similar order of magnitude in size in rodent and humans, scaling the absolute number of therapeutic cells homing to patients' tumors may be difficult to predict.

As experience with PFC labeling of cell therapy products grows, additional considerations may also be needed, for example, in the clinical batch scale-up of the labeling process [81] in specialized facilities. Furthermore, one could imagine having a cell therapy product expanded at a third-party site with a PFC label incorporated, and then shipped as a refrigerated or cryopreserved pre-labeled cell product; similar workflows are already in place for unlabeled, FDA-approved DC and CAR T cell products for cancer patients. Our view is that routine labeling of large cell batches can be engineered into a well-controlled process that can be exportable to multi-site clinical trials.

Additional logistical limitations to the development of routine fluorine imaging include the fact that clinical scanners are most often equipped for proton scans only. ${ }^{19} \mathrm{~F}$ MRI requires specialized detection coils and hardware modifications for image acquisition, which are not currently available in most MRI centers, but can be sourced by third parties $[82,83]$.

\section{Alternative cell detection strategies - Nuclear imaging}

The potential use of radionuclide-based imaging methods, particularly PET and SPECT, are an alternative to ${ }^{19} \mathrm{~F}$ MRI cell detection $[15,84]$. Generally, nuclear imaging methods have a high potential sensitivity in vivo. Detection of cells labeled with radioactive tracers ex vivo is feasible, but can be challenged by passive leakage of the radioactive tracer from labeled cells, potential radiotoxicity to cells, and a limited time window for scanning due to the limited half-life of the radioisotope. The use of radiolabeled leukocytes has precedent clinically for diagnostic inflammation detection. For example, Ceretec $^{\text {TwN }}$ (GE Healthcare), a SPECT labeling agent containing radioactive technetium-99 to label white cells ex vivo that are reinfused, is an FDA-approved diagnostic for intra-abdominal infection and inflammatory bowel disease.

Other nuclear imaging approaches employ gene reporters [85, 86]. Reporters require vector transduction of therapeutic cells prior to infusion. Subsequently, a radioactive substrate is infused systemically in vivo to enable imaging of transduced cells. This approach has the benefit of the potential for long-term detection of cell products that proliferate in vivo. Current PET tracers with potential for clinical cell therapy imaging include HSV-FIAU [87] and $\left[{ }^{18} \mathrm{~F}\right]$ F-Ara-G [88] reporters. Reporters require high-efficiency cell transduction manipulations and would not be practical for certain autologous cells like TILs. The ${ }^{18} \mathrm{~F}$ has a half-life of $\sim 110 \mathrm{~min}$ thereby limiting longitudinal studies from a single substrate dose.

Another alternative is PET diabody technology that uses antibody fragments against CD4 and CD8 receptors with ${ }^{89} \mathrm{Zr}$ or ${ }^{64} \mathrm{Cu}$ (half-lives 768 and $13 \mathrm{~h}$, respectively) resulting in specific targeting of T-cells in vivo $[89,90]$. This technology does not require ex vivo manipulation of the cells but does not distinguish between endogenous host cells and adoptively transferred cells in vivo [91]. Overall, cell quantification in situ using PET reporter and antibody-based approaches present several challenges to date but remain an emerging area of research.

\section{Conclusion}

Our view is that cell labeling is a well-controlled and validated process that has been reproduced by numerous laboratories. The properties of labeled cells, such as labeling levels (i.e., mean ${ }^{19} \mathrm{~F} / \mathrm{cell}$ ) and intracellular localization of PFC, are predictable based on intrinsic phagocytic tendencies, physical cell size, high-level function in the body, and cell activation status and health during the labeling process. Fluorine MRI enables noninvasive monitoring of in vivo survival and behavior of therapeutic cells, as well as their indirect effect on cancer cells. Overall, the use of ${ }^{19} \mathrm{~F}$-based MRI cell detection of cell therapy products in vivo is still in the early adaptor phase, but holds promise for advancing a wide range of cell therapy trials for cancer.

\section{Abbreviations \\ ACT: Adoptive cell therapy; CAR: Chimeric antigen receptor; CFSE: 5(6)- Carboxyfluorescein N-hydroxysuccinimidyl ester; DC: Dendritic cell; EGFRvIll: Epidermal growth factor receptor variant three; MHC: Major histocompatibility complex; MRI: Magnetic resonance imaging; NK: Natural killer; NMR: Nuclear magnetic resonance; PBMC: Peripheral blood mononuclear cells; PCE: Perfluoro-15-crown-5-ether; PET: Positron emission tomography; PFC: Perfluorocarbon; PFOB: Perfluorooctyl bromide; PFPE: Perfluoropolyether; SPECT: Single photon emission coherent tomography; TCR: T cell receptor; TIL: Tumor infiltrating lymphocyte}

\section{Funding}

CMC is supported by the National Institutes of Health $(\mathrm{NIH}) \mathrm{NCl}$ K08 CA174750, Carbone Cancer Center pilot grant NIH NCI P30 CA014520, the Stand Up To Cancer St. Baldrick's Pediatric Dream Team Translational Research Grant SU2C-AACR-DT1113, Hyundai Hope on Wheels and the MACC fund. Stand Up To Cancer is a program of the Entertainment Industry Foundation administered by the American Association for Cancer Research. Funding for ETA was provided by NIH grants R01-EB017271, R01-EB024015, R01 CA139579 and the California Institute for Regenerative Medicine grant LA1-C12-06919.

\section{Availability of data and materials}

Data sharing is not applicable to this article as no datasets were generated or analyzed during the current study.

Authors' contributions

FC performed the literature search and wrote the first draft of the manuscript. ETA and CMC edited the manuscript. All authors read and approved the final manuscript. 


\section{Ethics approval and consent to participate}

Not applicable.

\section{Consent for publication \\ Not applicable.}

\section{Competing interests}

ETA is a founder, member of the advisory board and shareholder of Celsense, Inc. FC and CMC have no relevant conflicts of interest to disclose.

\section{Publisher's Note}

Springer Nature remains neutral with regard to jurisdictional claims in published maps and institutional affiliations.

\section{Author details \\ 'Department of Bioengineering, University of California San Diego, 2880 Torrey Pines Scenic Drive, La Jolla, CA 92037, USA. ²Department of Pediatrics and Carbone Cancer Center, University of Wisconsin School of Medicine and Public Health, 1111 Highland Avenue, Madison, WI 53705, USA. ${ }^{3}$ Department of Radiology, University of California of San Diego, 9500 Gilman Dr. \#0695, La Jolla, CA 92093-0695, USA.}

Received: 13 June 2018 Accepted: 21 September 2018

Published online: 11 October 2018

\section{References}

1. Fine HA, Dear KB, Loeffler JS, Black PM, Canellos GP. Meta-analysis of radiation therapy with and without adjuvant chemotherapy for malignant gliomas in adults. Cancer. 1993;71:2585-97.

2. Dunn GP, Bruce AT, Ikeda H, Old L, Schreiber RD. Cancer immunoediting: from immunosurveillance to tumor escape. Nat Immunol. 2002;3:991-8.

3. Liu Y, Zeng G. Cancer and innate immune system interactions: translational potentials for cancer immunotherapy. J Immunother. 2012;35:299-308.

4. Borghaei H, Smith MR, Campbell KS. Immunotherapy of cancer. Eur J Pharmacol. 2009;625:41-54

5. Bubenik J. Tumour MHC class I downregulation and immunotherapy (review). Oncol Rep. 2003;10:2005-8.

6. Rosenberg SA, Packard BS, Aebersold PM, Solomon D, Topalian SL, Toy ST, et al. Use of tumor-infiltrating lymphocytes and interleukin-2 in the immunotherapy of patients with metastatic melanoma. A preliminary report. N Engl J Med. 1988;319:1676-80.

7. Dotti G, Savoldo B, Brenner M. Fifteen years of gene therapy based on chimeric antigen receptors: "are we nearly there yet?". Hum Gene Ther. 2009;20:1229-39.

8. Fesnak $A D$, June $\mathrm{CH}$, Levine BL. Engineered T cells: the promise and challenges of cancer immunotherapy. Nat Rev Cancer. 2016;16:566-81.

9. Conradi MS, Saam BT, Yablonskiy DA, Woods JC. Hyperpolarized 3He and perfluorocarbon gas diffusion MRI of lungs. Prog Nuc Magn Reson Spect. 2006:48:63-83.

10. O'Rourke DM, Nasrallah MP, Desai A, Melenhorst JJ, Mansfield K, Morrissette $J J D$, et al. A single dose of peripherally infused EGFRvIll-directed CAR T cells mediates antigen loss and induces adaptive resistance in patients with recurrent glioblastoma. Sci Transl Med. 2017;9:eaaa0984.

11. Larimer BM, Wehrenberg-Klee E, Dubois F, Mehta A, Kalomeris T, Flaherty K, et al. Granzyme B PET imaging as a predictive biomarker of immunotherapy response. Cancer Res. 2017;77:2318-27.

12. Au P, Hursh DA, Lim A, Moos MC Jr, Oh SS, Schneider BS, et al. FDA oversight of cell therapy clinical trials. Sci Transl Med. 2012;4:149fs31.

13. FDA. Guidance for industry, preclinical assessment of investigational cellular and gene therapy products. 2013. https://www.fda.gov/downloads/ biologicsbloodvaccines/guidancecomplianceregulatoryinformation/ guidances/cellularandgenetherapy/ucm376521.pd.

14. Ahrens ET, Helfer BM, O'Hanlon CF, Schirda C. Clinical cell therapy imaging using a perfluorocarbon tracer and fluorine-19 MRI. Magn Reson Med. 2014; 72:1696-701.

15. Kircher MF, Gambhir SS, Grimm J. Noninvasive cell-tracking methods. Nat Rev Clin Oncol. 2011:8:677-88.

16. FDA. Initiative to reduce unnecessary radiation exposure from medical imaging [https://www.fda.gov/radiation-emittingproducts/radiationsafety/ radiationdosereduction/default.htm].
17. Holland GNBPA, Hinshaw WS. 19F magnetic resonance imaging. J Magn Reson. 1977:28:133-6.

18. Bachert P. Pharmacokinetics using fluorine NMR in vivo. Prog Nuc Magn Reson Spect. 1998:33:1-56.

19. Flogel U, Ahrens ET. Fluorine Magnetic Resonance Imaging. Suntec: Pan Stanford Publishing Pte. Ltd.; 2017.

20. Janjic JM, Srinivas M, Kadayakkara DK, Ahrens ET. Self-delivering nanoemulsions for dual fluorine-19 MRI and fluorescence detection. J Am Chem Soc. 2008;130:2832-41.

21. Srinivas M, Morel PA, Ernst LA, Laidlaw DH, Ahrens ET. Fluorine-19 MRI for visualization and quantification of cell migration in a diabetes model. Magn Reson Med. 2007;58:725-34.

22. Janjic JM, Ahrens ET. Fluorine-containing nanoemulsions for MRI cell tracking. Wiley Interdiscip Rev Nanomed Nanobiotechnol. 2009;1:492-501.

23. Krafft MP. Fluorocarbons and fluorinated amphiphiles in drug delivery and biomedical research. Adv Drug Deliv Rev. 2001;47:209-28.

24. Riess JG. Understanding the fundamentals of perfluorocarbons and perfluorocarbon emulsions relevant to in vivo oxygen delivery. Artif Cells Blood Substit Immobil Biotechnol. 2005;33:47-63.

25. Partlow KC, Chen J, Brant JA, Neubauer AM, Meyerrose TE, Creer MH, et al. 19F magnetic resonance imaging for stem/progenitor cell tracking with multiple unique perfluorocarbon nanobeacons. FASEB J. 2007;21:1647-54.

26. Maa YF, Hsu CC. Performance of sonication and microfluidization for liquidliquid emulsification. Pharm Dev Technol. 1999;4:233-40.

27. Varescon C, Arlen C, Leblanc M, Riess JG. An easy, convenient way of describing the stability of fluorocarbon emulsions. J Chim Phys Biol. 1989;86:2111-7.

28. Lowe KC. Engineering blood: synthetic substitutes from fluorinated compounds. Tissue Eng. 2003:9:389-99.

29. Balducci A, Wen Y, Zhang Y, Helfer BM, Hitchens TK, Meng WS, et al. A novel probe for the non-invasive detection of tumor-associated inflammation. Oncoimmunol. 2013;2:e23034.

30. Stares E, Rho J, Ahrens ET, Foster P, Li A, Bartha R. Chapter 6 Fluorine-based contrast agents. In: Contrast agents for MRI: experimental methods. London: The Royal Society of Chemistry; 2018. p. 479-98.

31. Waiczies H, Lepore S, Janitzek N, Hagen U, Seifert F, Ittermann B, et al. Perfluorocarbon particle size influences magnetic resonance signal and immunological properties of dendritic cells. PLoS One. 2011;6:e21981.

32. Waiczies S, Niendorf T, Lombardi G. Labeling of cell therapies: how can we get it right? Oncoimmunol. 2017;6:e1345403.

33. Kabalnov AS, Shchukin ED. Ostwald ripening theory: applications to fluorocarbon emulsion stability. Adv Sci. 1992;38:69-97.

34. Ruiz-Cabello J, Walczak P, Kedziorek DA, Chacko VP, Schmieder AH, Wickline SA, et al. In vivo "hot spot" MR imaging of neural stem cells using fluorinated nanoparticles. Magn Reson Med. 2008;60:1506-11.

35. Srinivas M, Turner MS, Janjic JM, Morel PA, Laidlaw DH, Ahrens ET. In vivo cytometry of antigen-specific t cells using 19F MRI. Magn Reson Med. 2009; 62:747-53.

36. Somanchi SS, Kennis BA, Gopalakrishnan V, Lee DA, Bankson JA. In vivo (19)F-magnetic resonance imaging of adoptively transferred NK cells. Methods Mol Biol. 2016;1441:317-32.

37. Bouchlaka MN, Ludwig KD, Gordon JW, Kutz MP, Bednarz BP, Fain SB, et al. (19)F-MRI for monitoring human NK cells in vivo. Oncoimmunology. 2016;5: e1143996.

38. Bible E, Dell'Acqua F, Solanky B, Balducci A, Crapo PM, Badylak SF, et al. Non-invasive imaging of transplanted human neural stem cells and ECM scaffold remodeling in the stroke-damaged rat brain by (19)F- and diffusion-MRI. Biomaterials. 2012;33:2858-71.

39. Helfer $B M$, Balducci $A$, Nelson AD, Janjic JM, Gil RR, Kalinski $P$, et al. Functional assessment of human dendritic cells labeled for in vivo (19)F magnetic resonance imaging cell tracking. Cytotherapy. 2010;12:238-50.

40. Chapelin F, Gao S, Okada H, Weber TG, Messer K, Ahrens ET. Fluorine-19 nuclear magnetic resonance of chimeric antigen receptor $T$ cell biodistribution in murine cancer model. Sci Rep. 2017;7:17748.

41. Gonzales C, Yoshihara HA, Dilek N, Leignadier J, Irving M, Mieville $\mathrm{P}$, et al. In vivo detection and tracking of T cells in various organs in a melanoma tumor model by 19F-fluorine MRS/MRI. PLoS One. 2016;11:e0164557.

42. Alberts BJA, Lewis J, Alberts B, Raff M, Roberts K, Walter P. Lymphocytes and the cellular basis of adaptive immunity. Molecular biology of the cell. 4th ed. New York: Garland Science, a memeber of the Taylor \& Francis Group; 2002.

43. Ahrens ET, Flores $\mathrm{R}, \mathrm{Xu} H$, Morel PA. In vivo imaging platform for tracking immunotherapeutic cells. Nat Biotechnol. 2005;23:983-7. 
44. Hitchens TK, Liu L, Foley LM, Simplaceanu V, Ahrens ET, Ho C. Combining perfluorocarbon and superparamagnetic iron-oxide cell labeling for improved and expanded applications of cellular MRI. Magn Reson Med. 2015;73:367-75.

45. Patrick MJ, Janjic JM, Teng H, O'Hear MR, Brown CW, Stokum JA, et al. Intracellular pH measurements using perfluorocarbon nanoemulsions. J Am Chem Soc. 2013;135:18445-57.

46. Srinivas M, Boehm-Sturm P, Figdor CG, de Vries IJ, Hoehn M. Labeling cells for in vivo tracking using (19)F MRI. Biomaterials. 2012;33:8830-40.

47. Kalos M, June $\mathrm{CH}$. Adoptive T cell transfer for cancer immunotherapy in the era of synthetic biology. Immunity. 2013;39:49-60.

48. Anitei MG, Zeitoun G, Mlecnik B, Marliot F, Haicheur N, Todosi AM, et al. Prognostic and predictive values of the immunoscore in patients with rectal cancer. Clin Cancer Res. 2014;20:1891-9.

49. Fritzsching B, Fellenberg J, Moskovszky L, Sapi Z, Krenacs T, Machado I, et al. CD8(+)/FOXP3(+)-ratio in osteosarcoma microenvironment separates survivors from non-survivors: a multicenter validated retrospective study. Oncoimmunol. 2015;4:e990800.

50. Ahrens ET, Young WB, Xu H, Pusateri LK. Rapid quantification of inflammation in tissue samples using perfluorocarbon emulsion and fluorine-19 nuclear magnetic resonance. BioTechniques. 2011;50:229-34.

51. Waldhauer I, Steinle A. NK cells and cancer immunosurveillance. Oncogene. 2008;27:5932-43.

52. Lee SJ, Kang WY, Yoon Y, Jin JY, Song HJ, Her JH, et al. Natural killer (NK) cells inhibit systemic metastasis of glioblastoma cells and have therapeutic effects against glioblastomas in the brain. BMC Cancer. 2015;15:1011.

53. Poli A, Wang J, Domingues O, Planaguma J, Yan T, Rygh CB, et al. Targeting glioblastoma with NK cells and mAb against NG2/CSPG4 prolongs animal survival. Oncotarget. 2013;4:1527-46.

54. Dahlberg Cl, Sarhan D, Chrobok M, Duru AD, Alici E. Natural killer cell-based therapies targeting cancer: possible strategies to gain and sustain antitumor activity. Front Immunol. 2015;6:605.

55. Hermanson DL, Kaufman DS. Utilizing chimeric antigen receptors to direct natural killer cell activity. Front Immunol. 2015;6:195.

56. Banchereau J, Palucka AK. Dendritic cells as therapeutic vaccines against cancer. Nat Rev Immunol. 2005;5:296-306.

57. Figdor CG, de Vries IJ, Lesterhuis WJ, Melief CJ. Dendritic cell immunotherapy: mapping the way. Nat Med. 2004;10:475-80.

58. Dewitte H, Geers B, Liang S, Himmelreich U, Demeester J, De Smedt SC, et al. Design and evaluation of theranostic perfluorocarbon particles for simultaneous antigen-loading and (1) (9)F-MRI tracking of dendritic cells. J Control Release. 2013;169:141-9.

59. Ku MC, Edes I, Bendix I, Pohlmann A, Waiczies H, Prozorovski T, et al. ERK1 as a therapeutic target for dendritic cell vaccination against high-grade gliomas. Mol Cancer Ther. 2016;15:1975-87.

60. Kantoff PW, Higano CS, Shore ND, Berger ER, Small EJ, Penson DF, et al. Sipuleucel-T immunotherapy for castration-resistant prostate cancer. N Engl J Med. 2010;363:411-22.

61. Fink C, Gaudet JM, Fox MS, Bhatt S, Viswanathan S, Smith M, et al. (19)Fperfluorocarbon-labeled human peripheral blood mononuclear cells can be detected in vivo using clinical MRI parameters in a therapeutic cell setting. Sci Rep. 2018:8:590.

62. Garrelts JC. Fluosol: an oxygen-delivery fluid for use in percutaneous transluminal coronary angioplasty. DICP. 1990;24:1105-12.

63. Ilgiavishute la S, Zhukauskas G, Dapshis KL, Onishchenko NA. The use of perfluorocarbon emulsions for suppression of rejection reaction in kidney allotransplantation. Khirurgiia (Mosk). 1993:5:16-8.

64. Riess JG. Oxygen carriers ("blood substitutes")--raison d'etre, chemistry, and some physiology. Chem Rev. 2001;101:2797-920.

65. Sotak CH, Hees PS, Huang HN, Hung MH, Krespan CG, Raynolds S. A new perfluorocarbon for use in fluorine-19 magnetic resonance imaging and spectroscopy. Magn Reson Med. 1993;29:188-95.

66. Dardzinski BJ, Sotak CH. Rapid tissue oxygen tension mapping using $19 \mathrm{~F}$ inversion-recovery echo- planar imaging of perfluoro-15-crown-5-ether. Magn Reson Med. 1994;32:88-97.

67. Hees PS, Sotak CH. Assessment of changes in murine tumor oxygenation in response to nicotinamide using 19F NMR relaxometry of a perfluorocarbon emulsion. Magn Reson Med. 1993;29:303-10.

68. Mason RP, Antich PP, Babcock EE, Constantinescu A, Peschke P, Hahn EW. Non-invasive determination of tumor oxygen tension and local variation with growth. Int J Radiat Oncol Biol Phys. 1994;29:95-103.
69. Noth U, Morrissey SP, Deichmann R, Adolf H, Schwarzbauer C, Lutz J, et al. In vivo measurement of partial oxygen pressure in large vessels and in the reticuloendothelial system using fast 19F-MRI. Magn Reson Med. 1995;34: $738-45$.

70. Kadayakkara DK, Janjic JM, Pusateri LK, Young WB, Ahrens ET. In vivo observation of intracellular oximetry in perfluorocarbon-labeled glioma cells and chemotherapeutic response in the CNS using fluorine-19 MRI. Magn Reson Med. 2010;64:1252-9.

71. Zhong J, Sakaki M, Okada H, Ahrens ET. In vivo intracellular oxygen dynamics in murine brain glioma and immunotherapeutic response of cytotoxic T cells observed by fluorine-19 magnetic resonance imaging. PLoS One. 2013;8:e59479.

72. Ribot EJ, Gaudet JM, Chen Y, Gilbert KM, Foster PJ. In vivo MR detection of fluorine-labeled human MSC using the bSSFP sequence. Int J Nanomedicine. 2014:9:1731-9.

73. Khurana A, Chapelin F, Beck G, Lenkov OD, Donig J, Nejadnik H, et al. Iron administration before stem cell harvest enables MR imaging tracking after transplantation. Radiology. 2013;269:186-97.

74. Makela AV, Gaudet JM, Foster PJ. Quantifying tumor associated macrophages in breast cancer: a comparison of iron and fluorine-based MRI cell tracking. Sci Rep. 2017;7:42109.

75. Castro O, Nesbitt AE, Lyles D. Effect of a perfluorocarbon emulsion on reticuloendothelial system clearance function. Am J Hematol. 1984;16:15-21.

76. Ahrens ET, Bulte JW. Tracking immune cells in vivo using magnetic resonance imaging. Nat Rev Immunol. 2013;13:755-63.

77. Zhong J, Mills PH, Hitchens TK, Ahrens ET. Accelerated fluorine-19 MRI cell tracking using compressed sensing. Magn Reson Med. 2013;69:1683-90.

78. Kislukhin AA, Xu H, Adams SR, Narsinh KH, Tsien RY, Ahrens ET. Paramagnetic fluorinated nanoemulsions for sensitive cellular fluorine-19 magnetic resonance imaging. Nat Mater. 2016;15:662-8.

79. Ruiz-Cabello J, Barnett BP, Bottomley PA, Bulte JWM. Fluorine (F-19) MRS and MRI in biomedicine. NMR Biomed. 2011;24:114-29.

80. Stenzel M, Mentzel HJ. Ultrasound elastography and contrast-enhanced ultrasound in infants, children and adolescents. Eur J Radiol. 2014;83:1560-9.

81. O'Hanlon CF, Fedczyna T, Eaker S, Shingleton WD, Helfer BM. Integrating a 19F MRI tracer agent into the clinical scale manufacturing of a T-cell immunotherapy. Contrast Media Mol Imaging. 2017;2017:7.

82. Hu L, Keupp J, Caruthers S, Goette M, Lanza G. Wickline S: chapter 2 advanced detection techniques and hardware: simultaneous 19 F/ 1 H MRI. Suntec: Pan Stanford Publishing Pte. Ltd.; 2016.

83. Schmieder AH, Caruthers SD, Keupp J, Wickline SA, Lanza GM. Recent advances in (19)fluorine magnetic resonance imaging with perfluorocarbon emulsions. Engineering (Beijing). 2015;1:475-89.

84. McCracken MN, Tavare R, Witte ON, Wu AM. Advances in PET detection of the antitumor T cell response. Adv Immunol. 2016;131:187-231.

85. Gambhir SS, Barrio JR, Herschman HR, Phelps ME. Assays for noninvasive imaging of reporter gene expression. Nucl Med Biol. 1999;26:481-90.

86. Kang JH, Chung JK. Molecular-genetic imaging based on reporter gene expression. J Nucl Med. 2008;49(Suppl 2):164S-79S.

87. Koehne G, Doubrovin M, Doubrovina E, Zanzonico P, Gallardo HF, Ivanova A, et al. Serial in vivo imaging of the targeted migration of human HSV-TKtransduced antigen-specific lymphocytes. Nat Biotechnol. 2003;21:405-13.

88. Ronald JA, Kim BS, Gowrishankar G, Namavari M, Alam IS, D'Souza A, et al. A PET imaging strategy to visualize activated T cells in acute graft-versus-host disease elicited by allogenic hematopoietic cell transplant. Cancer Res. 2017; 77:2893-902.

89. Tavare R, Escuin-Ordinas H, Mok S, McCracken MN, Zettlitz KA, Salazar FB, et al. An effective immuno-PET imaging method to monitor CD8dependent responses to immunotherapy. Cancer Res. 2016;76:73-82.

90. Tavare R, McCracken MN, Zettlitz KA, Knowles SM, Salazar FB, Olafsen T, et al. Engineered antibody fragments for immuno-PET imaging of endogenous CD8 + T cells in vivo. Proc Natl Acad Sci U S A. 2014;111:1108-13.

91. Tavare R, McCracken MN, Zettlitz KA, Salazar FB, Olafsen T, Witte ON, et al. Immuno-PET of murine T cell reconstitution Postadoptive stem cell transplantation using anti-CD4 and anti-CD8 Cys-Diabodies. J Nucl Med. 2015;56:1258-64. 